\title{
Posicionamientos políticos de mujeres mapuche guluche: género, feminismos y razón colonial':
}

\author{
Political positions of Guluche Mapuche women: gender, feminisms \\ and colonial reason
}

Ximena Mercado-Catriñir

\section{Resumen}

En el contexto del resurgimiento de movimientos de mujeres y feministas entre el 20142018 en gulumapu ${ }^{2}$, se visibilizan casos de violencia contra mujeres al interior del movimiento social mapuche, dando cuenta de la necesidad de abordajes político-culturales propios para enfrentar estas situaciones. Esta investigación, mediante la implicación de epistemologías indígenas, mapuche y feministas descolonizadoras junto con metodologías, y procedimientos mapuche de discusión y análisis colectivo como güxamkan y güxamkawün, aborda los posicionamientos políticos de mujeres mapuche respecto al género, feminismo(s), las violencias externas e internas en la sociedad mapuche y perspectivas crítico deconstructivas respecto del fenómeno colonialismo-patriarcadocapitalismo y la minorización de estas problemáticas en los procesos autonómicos mapuche.

Palabras claves: Posicionamientos políticos, mujeres mapuche, violencias internas, feminismos indígenas, colonialismo internalizado.

\footnotetext{
Abstract

In the context of the resurgence of women's and feminist movements between 2014-2018 in ngulumapu, cases of violence against women are visible within the Mapuche social

* Doctorante en Ciencias Sociales de la Universidad de La Frontera, Temuco, Chile, ximena.mercadocatrinir@gmail.com

1 Participantes de los güxamkan (conversaciones) y, güxamkawün (encuentro/s) Carol Piutrin Quintrel, Cristel Malo Painen, María Moreno Raiman, Natividad Llanquileo Pilquiman, Isabel Cañet Caniulen, Janis Quinchavil Santis, Javiera Antil Fuentes, Ana Tragolaf Ancalaf y Paola Melita Guajardo.

${ }^{2}$ En referencia al territorio histórico mapuche que actualmente involucra Chile.
} 
movement, realizing the need for their own political-cultural approaches to address these situations. This research, through the involvement of indigenous, Mapuche, epistemologies decolonizing feminists together with Mapuche methodology and procedures for discussion and collective analysis such as güxamkan and güxamkawün, addresses the political positions of Mapuche women regarding gender, feminism (s), external violence and the internal violence in Mapuche society and its critical deconstructive perspectives regarding the colonialism-patriarchy-capitalism phenomenon and the minorization of these problems in the Mapuche autonomic processes.

Keywords: Political positions, Mapuche women's, internal violence, indigenous feminisms, internalized colonialism.

Fecha de recepción: Mayo 2021

Fecha de aprobación: Diciembre 2021

\section{Introducción}

Este artículo da cuenta de parte de los resultados de una investigación que se propuso comprender los posicionamientos políticos que mujeres mapuche, organizadas y vinculadas a procesos socioculturales, declaran en sus resistencias en torno a las violencias internas generizadas presentes en la sociedad mapuche. Fenómeno abordado en el contexto de las movilizaciones suscitadas en el período 2014-2018, momento en que el movimiento feminista toma lugar en distintos puntos del país, incluyendo la ciudad de Temuco -parte del territorio simbólico político mapuche wajmapu ${ }^{3}{ }^{4}$.

Abordar conjuntamente género y colonialismo en la cotidianeidad de la sociedad mapuche, involucra (re)situar el contexto colonial y la construcción de los estados-nación chileno y argentino, como evento que marca e impulsa un sucesivo proceso de transformación de las relaciones ecosociales y geopolíticas que constituían la base de esta sociedad, como nación originaria en su conjunto. Junto con ello, se hace necesario revisar las violencias coloniales impulsadas contra el ser y cuerpos de las mujeres, así como la trayectoria e implicación del constructo género, en dicho proceso colonial, configurando la transformación del lugar de las mujeres y otras corporalidades (Lugones, 2008) al interior del propio pueblo y respecto de occidente.

${ }^{3}$ Se adopta el grafemario wirilzugunwe, Universidad Católica de Temuco (Desiderio Catriquir y Gabriel Llanquinao)

${ }^{4}$ En referencia al territorio histórico mapuche compuesto por Gulumapu, Chile, y Puel Mapu, Argentina. Territorios que en la memoria mapuche se entiende como nación mapuche, cuya soberanía fue reconocida por la corona española y posteriormente negada por el estado moderno en Chile. 
La intervención colonial en wajmapu, como en distintos territorios de Abya Yala y el mundo, implicó para las mujeres un proceso de extrema y marcada violencia. Las violencias cruzadas que se siguen impulsando tuvieron dos expresiones marcadas. Una violencia frontal, contenida de una política de exterminio a razón de forjar un imperio y, posteriormente, un estado moderno colonial. Por otro lado, la violencia simbólica actúa interviniendo los códigos normativos del az mogen (normas de vida) y su orgánica.

La colonización consciente del valor trascendental de la espiritualidad impulsa intensivos procesos de evangelización en alianza con la instalación de fuertes y fundación de ciudades, que implicaron el ejercicio de violencia frontal o abierta. Proceso colonial y guerra con el imperio español que el pueblo mapuche resistió militar y políticamente, fijando un límite entre el dominio de jurisdicción española y el de jurisdicción mapuche, lo cual quedó estipulado en los parlamentos que sostenían ambas partes. Situación que, vuelve a punto cero a fines del siglo $X I X$, momento en que los ejércitos republicanos de Chile y Argentina vuelven a invadir estos territorios (Pichinao, 2015).

Este proceso remite históricamente a las mujeres originarias al lugar de la pasividad, sumisión y desprovisión de agencias necesarias para cuestionar-nos y modificar la condición de género al interior de la sociedad propia. Invisibilizando y minorizándose las múltiples dinámicas de participación de las mujeres indígenas al interior de los cuerpos colectivos la existencia de vínculos entre mujeres, como la potencia de pu kushe papay em (ancestras) al interior de las familias y territorios más allá de lo público, negándose y no comprendiéndose, desde esta óptica, la trama interactuante de opresiones sobre estos cuerpos en el proceso de intrusión civilizatoria (Cumes, 2012)

En este sentido, las mujeres como parte del colectivo enfrentan un nuevo proceso de usurpación territorial por parte del naciente estado nacional en Chile, el cual militarmente reduce el territorio controlado por los mapuche, de diez millones a quinientos mil hectáreas (Pichinao, 2015), situación que se acompaña del robo de kujiñ (ganado) que configuraban parte importante de la economía mapuche. Todo lo cual impacta profundamente en el mapuche mogen (sistema de vida mapuche) en el periodo post reduccional, estableciendo una larga trayectoria de carencias socioeconómicas y violencias colaterales que permean y cruzan la vida de la sociedad mapuche, resquebrando los tejidos societales, comunitarios y territoriales de pu lof, impulsando de esta forma procesos sucesivos de migración interna y desterritorialización forzada hacia los centros urbanos.

Así mismo, las diversas instituciones con que se forjaron los estados en Chile y Argentina, con sangre y miedo buscaron alejar a la sociedad mapuche de los conocimientos, prácticas y políticas propias, con el fin de negar, transformar y facilitar el avance de la conquista y fundación de un nuevo orden y estado nacional.

La razón colonial reproduce la estructura social de las familias fundantes del proceso colonial en la sociedad mapuche, forjando el "lugar de la servidumbre" a las "indias" (Cumes, 
2014) y chinas, y despojándolas de su saber, su hacer en la economía, el arte, la salud, la alimentación, como en distintas áreas que son fundamentales para la existencia de los pueblos, y que permitieron -y siguen permitiendo- que éstos sobrevivieran en tiempos de guerras y militarizaciones sucesivas. El resguardo de semillas, y conocimientos tradicionales han logrado mantenerse vigentes y recreándose gracias a la incansable acción de las mujeres, niñxs y ancianos, y no sólo de la fuerza de los hombres como sostiene el mito fundacional colonial. Esta situación se expresa en un fragmento de la historia de vida de Alicia citada en Nahuelpan (2015).

A nosotras nos enseñaban a contar, leer, a trabajar bien en la casa, porque decían que teníamos que servir bien nuestra casa, al marido, a los hijos, y después para buscar trabajo, para irse a trabajarle a los patrones. También se usaba harto lo de los mocitos, de los niños, las niñas que las familias entregaban a los gringos. Había una señora que tenía puras mocitas, chinitas para su servidumbre, como tres niñas crió esa señora, pero las niñas apenas podían irse, se iban escondidas. Porque las quería de puro esclavas no más. Las entregaban de chiquititas, yo conocí varias, las explotaban como animales, para eso las querían, porque ¿quién va cuidar lo ajeno? (p. 290)

De este modo, la patriarcalización colonial va calando en el mapuche mogen, extirpando la idea de ser persona contenida en la palabra che, imponiendo la masculinización del término, asociándosela al hombre y estableciendo categorías de femineidad y masculinidad y una relación separada de la organicidad que define al mapuche. A la vez que mina y reconfigura la vida de las mujeres y niñxs, como de las familia y territorios, respecto de la propia comprensión del cuerpo, la sexualidad, fecundidad y aborto, las decisiones y práctica de conformación de familias, el sentido de lo político, y el rol de las mujeres en la economía, las artes y los sistemas agroalimentarios ${ }^{6}$, como el valor político-espiritual que estas áreas poseen en la mantención y recreación de sociedades en resistencia.

La demarcación territorial y corporalidades mapuche implicó un intensivo proceso de violencia epistémica y espiritual, cuyos procesos aún no logran resolverse.

A partir de lo anterior, esta investigación se propuso profundizar en los aportes que las mujeres mapuche organizadas y activistas han forjado y proyectan en la descolonización del saber y el hacer respecto al género y al feminismo, y con ello, dar respuestas a las siguientes interrogantes: ¿Cuáles son los posicionamientos políticos de las mujeres mapuche organizadas y autónomas en torno a violencias internas generizadas al interior de

${ }^{5}$ Entrevista citada en Antileo, Cárcamo-Huechante, Calfio y Huinca-Piutrin. 2015, p. 290.

'Entre ello, el resguardo de semillas y la significación propia de la alimentación y la salud comprendida como fortalecimiento espiritual y por tanto vinculada a aspectos que trascienden el acto de comer. 
la sociedad mapuche?, ¿Cuáles son los mecanismos que se plantean para abordarlas y ¿Cómo se vincula el principio de dualidad y complementariedad con estos posicionamientos?.

Esta investigación abordó colectivamente las resistencias y epistemologías que sustentan los posicionamientos políticos de un grupo de identidades sociales específicas de mujeres mapuche, quienes (re)interpretamos la memoria y el devenir de sus cuerpos, y otros cuerpos marginalizados en relación con el cuerpo colectivo mapunche ${ }^{7}$ y su proceso de autodeterminación. Tales apuestas se dinamizan en un complejo contexto de contradicciones y dificultades, instaladas sucesivamente por el neocolonialismo y su internalización en el mapuche mogen (sistema de vida mapuche).

\section{Colonialismo, Género y Pueblo Mapuche}

En la memoria mapuche y historia no oficial chilena habita la guerra, la evangelización y la domesticación del "salvaje", y una historia de levantamientos por parte de los mapuche en contra de los conquistadores, permitiendo la victoria del mal llamado "desastre de Curalaba" que en 1598 se estableciera desde el río Bio Bío al sur como territorio independiente (Ríos, 2012; Bengoa, 2011) ${ }^{8}$, estableciéndose posteriormente relaciones comerciales y políticas entre ambas. A finales del siglo XIX y comienzos del siglo $\mathrm{XX}$, la validación de las autoridades tradicionales mapuche y los mecanismos de autoregulación propia como el az mapu, son anulados por las autoridades e instituciones del Estado colonial (Nahuelpan, 2013; Vásquez, 2015).

La instalación de la generización de las relaciones sociales propia de la colonialización se constituye en un proceso que irrumpe en las relaciones sociales y comunitarias, pues aún cuando la categoría teórica género emerge a partir de los años sesenta, el fenómeno tiene una larga data, y es sino hasta décadas posteriores que a partir de los feminismos negros y mujeres indígenas se devele el sentido racista, hegemónico y de ocultamiento de la brutalización, el abuso y la deshumanización que la colonialidad del género implica (Lugones, 2008, p. 82), así como, la comprensión monista de la estructura social y de las experiencias de dominación (Cumes, 2012).

Respecto al género y el patriarcado al interior de los pueblos indígenas y negros, autoras como Lugones, 2008; Espinosa 2009, Segato, 2011, entre otras, los refieren como

\footnotetext{
7 Término del pueblo mapuche que refiere a persona mapuche, quién junto con poseer dicha identidad, en razón del ordenamiento social y espiritual que rige a la sociedad mapuche, se le asocia una práctica y arraigo vinculado al ejercicio de su identidad en lo espiritual y lo cotidiano. Diferenciándose a partir de aquello, de la simple posesión de la calidad de ser o poseer dicha identificación.

${ }^{8}$ Citado en Peña, Huentequeo y Cayupan, (2018). Relatos de mujeres mapuche de la Araucanía: revisitación a las formas de familia bajo la figura de la bigamia. Tabula Rasa, (28), 323-346
} 
resultados del colonialismo europeo. Existen en este sentido distintas miradas respecto al patriarcado y el género al interior de los pueblos precoloniales, como es la inexistencia del género en el mundo precolonial (Lugones, 2007; Cumes, 2012; Oyěwùmí, 1997, 2017), o reconociéndolo como un principio organizativo fundamental de las sociedades occidentales, la existencia de nomenclaturas de género en las sociedades tribales y afro-americanas como el patriarcado de baja intensidad (Segato, 2018), patriarcado originario ancestral (Cabnal, 2010) o el entronque patriarcal (Paredes y Guzmán, 2014). Cumes (2012), por otra parte, aborda el patriarcado y el colonialismo como coparte del sistema de dominio, los que inciden en las sociedades indígenas y no indígenas, por lo que es necesario observar críticamente el presente de las sociedades originarias, sus procesos históricos, sus resistencias y cómo éstas afectan las nociones de dualidad, cuatricidad, la participación y dignidad de las mujeres. En estas últimas categorizaciones se identifican los movimientos de mujeres indígenas y feministas vinculadas al proceso de movilización social política en Chiapas y al proceso de los 500 años de colonización.

\section{Feminismos, movimientos de mujeres latinoamericanos e indígenas y nuevas epistemologías para sentipensar las realidades}

Las tensiones históricas respecto a la violencia de género han traído la proposición de otras categorías como "insterseccionalidad" (Crenshaw; 1991 2012) o fusión de opresiones vinculadas al racismo, clasismo y sexismo, para dar cuenta de la trama de violencias que afectan la vida de identidades colectivas racializadas, en cuyo caso el capitalismo y sus prácticas se entienden parte intrínseca del colonialismo (Grosfoguel, 2011).

Como una manera de enfrentar esta problemática desde las mujeres indígenizadas y racializadas, se argumentan contribuciones claves para el desarrollo del análisis feminista, situación que ha involucrado enfrentar una serie de cuestionamientos provenientes de los sectores increpados como de los propios círculos sociales, entre ellos compañeros y autoridades indígenas, quienes muy a menudo descalifican las demandas de las mujeres como solicitudes externas que no corresponden a la realidad de las comunidades indígenas (Hernández 2001, Espinosa 2009). Ello ha implicado una lenta escucha y validación del derecho a una vida libre de violencia, tanto en el derecho positivo como consuetudinario, constituyéndose esta última instancia de resolución de conflictos en un escenario que expresa cierta tendencia a dar lugar a justificaciones a los hechos e inculpación de las mujeres por sus roles no cumplidos, más que a validar la necesidad de resolver efectivamente tales problemáticas (Hernández 2001, Espinosa, 2009; Mercado, 2011; Salgado, 2012; Chirapaq y Ecmia, 2013, De Sousa Santos, 2012 entre otros). Situación que se propuso abordar esta investigación situada en gülumapu. 
Entre otros aportes surgidos a partir de la denuncia de las mujeres indígenas, es la instalación de la categoría violencia sexual en contexto de conflictos armados, la cual permite reconocerla como práctica de larga data, afín al proceso de colonización, donde las mujeres pasan a ser consideradas "botín de guerra, prisioneras que además de ser usadas como esclavas, las prestaciones sexuales entran a formar parte de su trabajo, sellando mediante este acto simbólico en situaciones de guerra, la conquista y el sometimiento de un pueblo" (Carrillo, Zapata y Vásquez; 2009, p. 135). Traduciéndose en la conformación de lo que Segato (2018) denomina como "fratria masculina", estructura corporativa que se desenvuelve en un contexto de impunidad y de internalización de "pedagogías de la crueldad", como referencia a los distintos actos y prácticas que enseñan, habitúan y programan a los sujetos a cosificar la vida.

Las pedagogías de la crueldad son relevantes de situar para comprender cómo la relación entre los estados y los múltiples extractivismos, junto con constituirse en un mecanismo que se configura en parte de las políticas de usurpación y violencia colonial, incorporan mecanismos simbólicos mediante los cuales infunden el miedo, los feminicidios -por causas de defensa territorial y ambientalismo-, y la naturalización y reproducción de su mal desarrollo, y la dinámica de violación de la vida en su integridad y orgánica por un proceso dinámico de explotación, desigualdad, injusticia y violencia (Shiva, 1995). Remirar esta relación desde la descolonización del conocimiento implica, entre otros aspectos, desmontar la matriz epistemológica de la modernidad occidental, capitalista y patriarcal reestablecida por los estados nacionales y las élites reproductoras de la colonización (Lugones, 2008).

\section{Descolonizar para sanar. Resistencias de mujeres mapuche y feminismos situados}

Desde las mujeres mapuche argumentamos otras expresiones no reconocidas ni visibilizadas en torno a violencias de género, -las que se entienden en una relación de alianza y confrontación en desmedro de la dignidad de los cuerpos generizados y minorizados, la violencia externa, que ha implicado distintos procesos de (des)encuentros y arbitrios con el Estado y la sociedad chilena, en su proceso tendiente a una interculturalidad crítica que tiende a desmoronarse; y la violencia interna (Mercado, Zegers, Tragolaf y Contreras, 2015), espacio de lo propio que, en lugar de constituir un espacio de refugio, pasa a ser otra vía de resistencias ante distintos obstáculos, en razón de los cuerpos que se habiten. Situación que delimita la necesaria descolonización interna y los procesos tendientes a la sanación del traumatismo histórico (Mitchell, 2005) con el fin de sanar y fortalecer las resistencias mapuche.

Como plantea Calfio (2012), las mujeres mapuche en este contexto pasan de tener y ejercer poder de decisión a ver no sólo reducidos los territorios al ámbito de la huerta, sino también integrar la mayor invisibilidad y violencia que se haya vivido colectivamente. 
Constituyendo la desvaloración por ambas sociedades un entorno complejo para la vida de las mujeres.

Despatriarcalizar los privilegios que el colonialismo y su trama patriarcadocapitalismo asienta sobre los cuerpos de los hombres reproduce e incorpora nuevas dificultades a quienes resisten y cuestionan sus prácticas. Entre ello, estigmatización, sanción social y, en algunos casos, la expulsión de los territorios bajo la excusa de ser convocantes, propiciadoras de lo foráneo, de la segregación social y quiebre de los procesos de resistencia (Quiñimil, 2012, Mercado et al; 2015, 2019) y del refuerzo de la intrusión colonial o awiñkamiento ${ }^{9}$. Situación que no ocurren cada vez que líderes hombres adhieren a partidos políticos, orgánicas de izquierda, dejan de usar su takuluwün ${ }^{10}$, asumen cargos en instituciones estatales y/o ejercen prácticas no propias del mapuche mogen ${ }^{11}$. Situación cuestionable en tanto un pueblo en resistencia debiese estar dispuesto a su dinamismo e incluir cambios que aporten a dicho fortalecimiento.

La violencia externa o estructural es ejercida desde el estado, por parte de los proyectos políticos gobernantes y transnacionales en desmedro de un pueblo y su territorialidad (Mercado; et al, 2015). Estos sostienen sucesivos procesos de militarización y criminalización de los procesos de defensa y control territorial, junto con la instalación de mecanismos tendientes al divisionismo u oposición interna entre quienes asumen vías distintas de exigibilidad de lo usurpado, gestan además fórmulas únicas para prevenir y abordar la violencia contra las "mujeres", despolitizándola de su carácter plural, político e histórico. Configurándose políticas públicas neocoloniales que se traducen en la aplicabilidad parcelada de los tratados internacionales en materia de derechos de las mujeres y pueblos indígenas.

Todo lo cual imposibilita la gestación de una interculturalidad crítica al interior de las políticas públicas en Chile, donde la apelación de los derechos de las mujeres supere el análisis aislado de la violencia basada en el género. Casos como la criminalización de una Machi $^{12}$, la desatención de su ejercicio espiritual y cultural por parte de MinMujeryEG ${ }^{13}$ y Sernameg ${ }^{14}$, la violencia obstétrica ejercida por Gendarmería en contra Lorenza Cayuhan ${ }^{15}$;

\footnotetext{
${ }^{9}$ Categoría mapuche asociada al inculpamiento de "traer ideas externas" al momento que mujeres mapuche sostenemos posicionamientos críticos para mirar y repensar lo propio en relación a la situación de las pu zomo o mujeres mapuche y otras corporalidades.

10 Vestimenta tradicional mapuche

11 Sistema de vida mapuche

12 Proceso que inicia a partir de la persecución política de la Machi Francisca Linconao Huircapan, una vez que gana un fallo contra el Estado, mediante el convenio 169 de la OIT, en el cual denuncia la tala ilegal de árboles y arbustos nativos de valor medicinal y espiritual por parte de un particular.

${ }^{13}$ Ministerio de la Mujer y Equidad de género

${ }^{14}$ Servicio Nacional de la Mujer y Equidad de género

15 Quién los días 12 y 13 de octubre del año 2016, a raíz de encontrarse en prisión preventiva por causas asociadas a procesos de restitución territorial mapuche, y producto de presentar molestias de salud vinculadas a su embarazo de 8 meses, es derivada de urgencia al Hospital de Concepción.
} 
el feminicidio irresuelto de Macarena Valdés por parte de Fiscalía ${ }^{16}$ y de Margarita Ancacoy ${ }^{17}$, quén fallece producto de un asalto en el trayecto de su casa a su lugar de trabajo, donde se desempeña en condición de subcontratación, entre otros casos que develan el sostenimiento de enfoques comprensivos segregados, la precarización y racialización de los derechos laborales y el acceso a la justicia en Chile por parte de las políticas públicas en materia de género.

Todo este marco de violencia estatal y violencias externas, por tanto, instala la necesidad de comprender que el cuerpo no es solo un espacio de interiorización de traumas, sino también de resistencias (Quiñimil, 2012). En este proceso los distintos saberes ancestrales no solo han sido objeto de violencias, sino también ejercidos y recreados continuamente, por tanto, debiesen posicionarse también las prácticas y saberes asociados al cuerpo de las mujeres como parte de los reclamos de autodeterminación de nuestro pueblo. Aspecto que mujeres mapuche, feministas y no, han venido instalando desde distintos planos de lo político, incluyendo el teórico y la micropolítica (MercadoCatriñir, 2011, 2019, 2020; Quiñimil, 2012, 2015; Calfio, 2012 Moreno, 2020).

\section{AzMapu. Sistema de autoregulación social o Justicia propia en la sociedad mapuche}

Las inquietudes que se asientan respecto a las violencias internas, entramadas con las violencias externas, constituirían para determinados sectores de mujeres mapuche, no sólo un problema, sino una salida para abordar la descolonización integral sanando y reparando toda la violencia generizada que se ha asentado sobre los cuerpos de las mujeres y niñxs y, por ende, en un pueblo-nación. En cuyo caso, el derecho propio mapuche, entendido como:

Tanto al momento de su traslado del Hospital de Arauco a Concepción como en su trabajo de parto y cesárea Gendarmería la mantiene engrillada. Caso que finalmente es llevado a la corte donde se resuelve a favor de los derechos de la demandante. Para mayores antecedentes del caso, consultar en https://bibliotecadigital.indh.cl/handle/123456789/1005

16 Dicho deceso ocurre el 22 de agosto de 2016 en el contexto de un proceso de defensa territorial, lugar en que la empresa transnacional RP Global se propone instalar una central de paso. Por parte del Servicio Médico Legal se argumentó la tesis de un suicidio, sin embargo los análisis forenses no coinciden con dichos antecedentes. Pese a lo cual, a la fecha el caso sigue sin resolverse. Para mayores antecedentes del caso, consultar en https://radio.uchile.cl/2020/08/21/cuatro-anos-sinjusticia-para-macarena-valdes-familia-y-organizaciones-continuan-sosteniendo-un-femicidioempresarial//

${ }_{17}$ Fallece alrededor de las 5 am, producto de un ataque perpetrado por tres jóvenes en el trayecto a su lugar de trabajo ubicado al interior de una universidad pública. Lugar en que desarrolla labores de limpieza en condiciones de subcontratación laboral. Mayores antecedentes en https://www.ciperchile.cl/2018/07/10/mujeres-invisibles-el-submundo-del-aseo-que-revelo-lamuerte-de-margarita-ancacoy/ 
El azmapu expresa las normas de conductas tanto individual y colectiva establecidas por la divinidad, que debe observar el mapuche para mantener la armonía cósmica. Estas normas deben ser asumidas por el hombre (che), en su relación primero con el mapu (Wajmapu y Wajontumapu), y luego con las personas entre sí, de manera de atender a la filosofía de vida (mogen) de nuestra cultura, ya que el derecho como producto de ésta, se encuentra estrechamente conectado y relacionado con la educación, con la religión, con la economía, con estructura política, etcétera, lo que se traduce en una cultura espiritual, en que todas las manifestaciones de ésta, están entrelazadas e incorporadas en la conciencia individual y colectiva del mapuche, en tanto pueblo-nación. (Maureira y Quidel, 2003: p. 1122)

La internalización del sistema patriarcal colonial como proceso que cala a fondo al interior del cuerpo colectivo mapuche constituye un llamado a descolonizar los sistemas propios y a efectivamente constituir en práctica la espiritualidad y perspectiva cosmobiocéntrica propia, y la orgánica interconectada e interdependiente que la configura. Se trataría por tanto de desbaratar lo que (Millaleo, 2012, citada en Quiñimil, 2012) refiere como el policía presente en cada uno de nosotros, el cual anula toda diferenciación por comprenderla como amenaza del orden y la moral mapuche. Situación que junto con ser una problemática, puede en sí misma ser una salida a la normalización impuesta desde el Estado.

En este sentido, como plantean Maureira y Quidel (2003), el az mapu constituye un sistema de autorregulación interna, un sistema ético que se traducen en códigos normativos territoriales orientados a establecer una noción y práctica de justicia restaurativa. Proceso que además debiese tener presente el momento histórico del que se se hace referencia ${ }^{18}$, entendiendo que las sociedades son dinámicas y humanas, no inmunes a las múltiples transformaciones y fenómenos sociales.

Las debilidades que manifiestan estas áreas del mapuche mogen y su organicidad, como plantea De Sousa Santos (2012), se arguyen como una estrategia desde los estados, que busca poner en evidencia las debilidades de los procesos autonómicos, razón por la que la impartición de justicia en materia de violencias internas al ser considerados como de menor cuantía o "pequeños litigios que al Estado le interesa reconocer o tolerar". Constituyendo la impartición de justicia en estas materias una vía de tensión de alta complejidad, en tanto que los derechos de las mujeres pasan a constituirse en una vía argumentativa de los estados para ir en contra de los procesos autonómicos, y a su vez, una problemática minorizada y postergada en ambos sistemas de justicia, consuetudinaria y ordinaria (Espinosa, 2009, Mercado, 2011; Salgado, 2012; Chirapaq y Ecmia, 2013; De Sousa Santos, 2012 entre otros).

\footnotetext{
${ }^{18}$ Relato participante en nvxamkawvn, citada en Mercado, Zegers, Contreras y Ancalaf, 2015.
} 
Un caso que grafica esta situación es la aplicación del derecho consuetudinario a que refieren los artículos 8 y 9 del convenio 169, como mecanismo de resolución de casos de violencia intrafamiliar, a partir de lo cual se validaron las disculpas públicas como un argumento válido para fallar dichos casos. La situación no fue sometida a consulta por parte de las mujeres ni del pueblo mapuche, y fue justamente lo que se propuso abordar una investigación realizada el año $2015^{19}$, donde se evidenció, desde mujeres mapuche, un profundo rechazo a este proceso, como al racismo que dejó entrever.

\section{Principio ético dualidad y complementariedad en la sociedad mapuche. Tensiones y desafíos}

El mundo espiritual y la sexualidad anterior a la intrusión colonial, el cristianismo y su razón, daba cuenta de otras prácticas y sentidos. Retazos de realidad que han podido reconstruirse a partir de crónicas de los colonizadores, y transmisión de la memoria oral de los kuifikecheyem (antepasadxs) y observaciones del mundo espiritual y natural por parte de kimche (sabixs). Comprensiones de mundo que comparten el sentido de que todo tiene vida y espíritu, constituyendo el plano espiritual un estado de equilibrio entre el ser humano y su entorno, donde la presencia femenina no es secundaria, sino implícita a la naturaleza y su abundancia (Sánchez, 2016).

En este contexto, la violencia espiritual (Chirapaq y Ecmia, 2013), como mecanismo de disciplinamiento cultural y de expresión de un sentido paternal ficticio que esconde formas de violencia para "salvar almas" e integrarlas a un modelo de lo esperable (Rivera, 2010), se constituye en un medio interventivo y transformador de las significaciones y aplicabilidad de los principios asociados a la coexistencia propios de las naciones/pueblos originarios, como es el principio ético de dualidad y complementariedad en la sociedad mapuche. Constituyendo la evangelización uno de los ejes de adoctrinamiento de seres autónomos al rol de servidumbre, inquilinaje y formas de esclavizaje abierto y encubierto. Proceso del cual no fueron ajenas otras mujeres, como las monjas, colonas y mestizas, y más complejo aún la propia sociedad, en los casos de Ixs niñxs kuñifal (huérfanos de padre, madre o ambos).

Una de las discusiones al interior de algunos movimientos y/o feminismos de mujeres indígenas es la carga heterosexual que posee esta comprensión de la vida, y su inaplicabilidad en la cotidianeidad social. Siendo una posible salida a esta discusión el continuar profundizando el análisis del kimün contenido en el mapuche feyentun.

\footnotetext{
19 Investigación social denominada "Mujeres mapuche y aplicación del convenio 169 en casos de Violencia Intrafamiliar en la Provincia del Cautín: ¿Reconocimiento del Derecho Propio Mapuche o deslegitimación de los derechos individuales de las mujeres mapuche?" desarrollada el año 2015, con metodologías participativas, con mujeres mapuche lideresas y vinculadas a organizaciones mapuche en la Región de la Araucanía, Chile.
} 


\section{Método}

Esta investigación se enmarca en la teoría crítica, considerando el carácter político con que tiñe la acción de investigar y al rol de co-construcción en que sitúa a Ixs sujetxs; en cuyo contexto las epistemologías descolonizadoras me permitieron optar por una alineación básica con las mujeres en resistencia y compromiso por producir conocimientocrítico.

En este contexto, "las" vidas de los pueblos indígenas no se asumieron en contenidos de la investigación, sino en paradigmas y métodos de hacer investigación reconocedores de las formas en que los propios pueblos indígenas crean y recrean conocimiento (Dei, 2013),

Las participantes de esta investigación fueron mujeres mapuche de la Región de la Araucanía, organizadas o autónomas, con prácticas claves respecto a la visibilización y abordaje de las violencias internas, siendo los criterios de inclusión: Mujeres mapuche mayores de 18 años, que no se encontraban en tratamiento actual por situaciones de violencia, ejercían roles de participación en procesos de defensa y visibilización de los derechos de las mujeres y habitaban en la región de la Araucanía, con experiencia de trabajo activo en $\operatorname{lof}^{20}$.

${ }^{20}$ En referencia a los territorios que componen wajmapu, la cual constituye una forma de or ganización socioterritorial específica. Otra forma de organización que se consideró para tales efectos, es la participación en comunidades indígenas, forma de organización y administración del territorio instaurada por el Estado. 
Revista Punto Género N. 16 Diciembre de 2021

ISSN 2735-7473 / 312- 337

Tabla №1: Participantes

\begin{tabular}{|l|l|l|l|}
\hline Códigos & Ocupación actual & $\begin{array}{l}\text { Tuwün21/ Autoidentificación } \\
\text { territorial }\end{array}$ & Edad \\
\hline NTR & Facilitadora intercultural & Wenteche & 51 \\
\hline NQX & Profesional & Wenteche & 32 \\
\hline NAF & Estudiante y laborante & Wenteche & 35 \\
\hline NPR & Estudiante & Bafkenche & 26 \\
\hline NCM & Profesional & Wenteche & 34 \\
\hline NMCH & Profesional independiente & Wenteche & 32 \\
\hline NMK & Estudiante de pregrado en receso & Bafkenche & 26 \\
\hline NLILI & Profesional & Lafkenche & 35 \\
\hline NAW & Profesional & Wenteche & 29 \\
\hline NMN & Profesional independiente & Champurria & 42 \\
\hline
\end{tabular}

Fuente: Elaboración propia

Las técnicas de construcción de datos, en coherencia con las perspectivas de las epistemologías indígenas y críticas, involucraron el uso de las técnicas güxamkan, o estilo de conversación que, considerando el procedimiento mapuche, implicaron el desarrollo de conversaciones personalizadas en base a una pauta semiestructurada y la elaboración de un consentimiento informado. Junto con el desarrollo de una instancia de güxamkawün, o encuentro ampliado de conversación entre las participantes, lo que favoreció el dialogo y discusión colectiva entre las participantes e investigadora en torno a los resultados arrojados inicialmente. Situación que aportó en la saturación y validación de los resultados preliminares. Ambas metodologías implicaron un protocolo previo de acercamiento e invitación al proceso, existiendo en varios de los casos vínculos previos a raíz de puestas políticas en común respecto a resistencias y acciones personales-colectivas en razón de la identidad y vinculación político espiritual como mapuche-zomo (mujeres-mapuche) en defensa del entramado que constituye a la sociedad mapuche.

Para el análisis de los datos, se consideró pertinentes los procedimientos analíticos desde los enfoques biográficos-narrativos: sobre violencias internas y externas y posicionamientos de mujeres mapuche respecto a traumatismo histórico y resistencias al respectoentre los años 2014-2018.

${ }^{21}$ Procedencia territorial 


\section{Resultados de la Investigación}

Los posicionamientos políticos se comprenderán parte de voces plurales dentro de la heterogeneidad característica de un cuerpo colectivo, como es la sociedad mapuche, la cual se entiende como dinámica y diversa. Entre los que se identificaron coincidencias y diferencias políticas a partir de trayectorias personales, territoriales y organizacionales. Se organizan en dos grandes ejes diferenciados y en vinculación conflictuante: Posicionamientos respecto a violencias externas y las violencias internas respectivamente.

\section{Posicionamientos respecto a las violencias externas}

Uno de los posicionamientos sobre los que se instalan las narrativas y posicionamientos políticos es que en las violencias externas se comprende la configuración de un eje relacional que marca la memoria y cotidianeidad del pueblo/nación mapuche y los distintos cuerpos-territorios que lo habitan:

"...la violencia viene de antes, ya sea violencia física o de menospreciar a alguien, viene de ese tiempo de las guerras, porque al final nosotros ante el español éramos seres absolutamente inferiores, y así, a medida que ha pasado el tiempo, siempre hemos sido seres inferiores, hemos visto que ese ser inferior trata a otro como inferior para no sentirse tan mal. Entonces, en este caso nosotras siempre hemos sido los seres inferiores en ciertos lugares. Los niños son sumamente maltratados por las monjas y todos esos traumas van quedando, y van quedando y después llega un cierto tiempo en que es la única emoción que no hemos podido controlar es la rabia y la frustración porque es algo que viene de atrás (...) mi papá desde los nueve años empezó a trabajar y ni siquiera iba a su casa, sino que era de estos niños que los arrendaban (..) nunca supimos como los maltrataron. Mi mamá, por otro lado, sumamente violentada en la escuela con las monjas. Entonces viene toda una historia de violencia y más las guerras, entonces ahí un poco uno puede entender de donde viene tanto, de donde viene tanta violencia" (NPR).

En este relato como en otros que confluyeron en estos diálogos, se plantea la violencia como un fenómeno histórico y sistemático que marca y acompaña la vida de quienes nos asumimos como mapunche, es decir todo quién práctica y ejerce la vida según los principios espirituales y de comprensión de la vida de la sociedad mapuche, en resistencia al actual modelo económico-político que impera en el mundo. Resistencia que se expresa en el distanciamiento y la asunción de una perspectiva critica respecto a las transformaciones que la intervención forzada del cristianismo ejerce al interior de la vida de las mujeres y los tejidos comunitarios:

"Como que ahora el tema del cristianismo siento que es súper potente como cambia la mentalidad de las formas de relacionarse en temas de familia, termina 
siendo súper coercitivo igual, súper autorepresivo de las mujeres, el tema de la sexualidad al menos, de que no se hable. (...) como que ahora se individualiza más todo y ahora con el sistema económico mucho más, mucho más. Es una realidad que en la comunidad se ha perdido esto tan comunitario que había antes" (NMP).

En ese sentido, las violencias externas, junto con comprenderse como un eje transversal que cruza las vidas de esta sociedad, se constituyen en un mecanismo que se internaliza y se reproduce colateralmente en contra de tus pares, y, por ende, es una problemática-acción relevante de abordar:

"A mí me preocupa lo que pasa entre nosotros, como que lo que el estado pueda hacer yo lo espero, y me puedo preparar para eso. Pero un hermano que no sepa que golpea a su hermana, que la maltrata en otra forma, que no procura cuidar a sus hijos, que ya no se sostiene el esto ha sido siempre" (NQI)

Un "esto ha sido siempre" que replantea la necesidad de profundizar en la referencia de tiempo que se habla para normalizar y acallar un problema instalado, como desde dónde emerge tal posición. Situación que conecta con la instalación e internalización de las violencias internas.

\section{Instalación e internalización transversal, reproducción y transformación de las violencias internas}

Uno de las situaciones problemáticas, transversal a la sociedad mapunche es la desatención, abandono y generización de la crianza y responsabilización de los niñxs, Siendo las mujeres y madres específicamente en quienes descansa esta responsabilización, situación que irrumpe en la noción de mapuche feyentun:

"Este aborto masculino de hijos e hijas, y que al parecer también en nuestro

pueblo existe desde que el tiempo es tiempo, hay palabras de hecho en mapuzungun para denominar ese acto, por lo tanto, ¡siempre existió! (NQI)".

Tal como refiere la cita anterior, junto con expresar una profunda critica respecto a la problemática situación actual que enfrenta las labores de la crianza y la niñez, queda la tensión de la aplicabilidad de los principios éticos y la justificación que en ésta recae para no reparar el daño:

<ya no se sostiene "no somos un pueblo machista, somos un pueblo dual" con gente joven. Ni siquiera estoy hablando de gente mayor, no? que ya tiene su estructura y así lo cree..> (NQI). 
"Esto siempre ha sido asi" a la vez que se asocia a una referencia desconectada del sentido propio del mapuche feyentun, el refuerzo de lo estático o monolítico como de la masculinización de la vida:

"...yo encuentro que la dualidad física, por ejemplo, es eso, complementarse, que no necesariamente tiene que ser entre un hombre y una mujer, porque espiritualmente uno no habla de un hombre y una mujer, sino que habla del ser, habla de un espíritu, el püjü" (NMK)

En este sentido, las criticas apuntan no sólo a un malestar personal o actual, sino a la conjugación de la memoria histórica, de a lo menos los últimos 200 años, y la realidad social que hoy dinamiza la cotidianeidad mapuche de distintos cuerpos-territorios:

"Bueno nosotras venimos de un contexto, allá en la comunidad, personalmente, de mi mamá eran 7 hermanas (...). En la cual la mujer era súper poco valorada, por lo mismo a mi abuelo siempre lo trataron mal, menos validado que los otros hombres. A pesar de eso, él sacaba a trabajar a sus hijas como cualquier hombre más del campo. (...) mi mamá es mamá soltera (...), se quedó al cuidado de los abuelos e igual, la misma historia. Súper poco valorada por el hecho que no estuviera casada, que no tuviera hijos hombres (...) siempre el comentario porque nunca se casaba, por qué nunca tuvo un hijo y ya cuando nacieron los nietos, recién como que adquirió otro valor en la comunidad, distinto era el trato, y eso una lo veía igual con otras mujeres que igual eran madres solteras, pero de hijos, tenían hijos. Entonces, siempre decían ya tiene el hijo que la cuide, quien la proteja, trabaje la tierra. Distinto con la niña, como era con nosotras..." (NPR)

En esos procesos, sin embargo, no están todas las mujeres haciéndose parte detal deconstrucción, sino que algunas de ellas asumen complicidades abiertas o silenciosas, tal como ocurre en distintas sociedades, como efecto de la patriarcalización de un cuerpo colectivo:

$<$...yo creo que hay mucho miedo igual de quemarse, de que te van a llamar resentida, alborotadora, supongo que acá el proceso será mucho más lento que en otros lados. Esto debiese tener un lugar súper importante, obvio, pero no va a pasar si las propias mujeres no lo declaran. Así como: "esto es así!", como que el tema de negarlo permite que se siga perpetuando, es complicidad!> (NMP).

Negación, aminoración y/o justificación del mal actuar que va en detrimento del kümefelen (bien estar) de sí mismx, sus pares y niñxs implicados en tales casos, como un pueblo-nación en su conjunto:

"...cuando llega el estado chileno (..) particularmente son los hombres que lideran ese proceso de guerra, de lucha y son, también en el fondo, luchas de poderes que también se dan ahí (...) y bueno ahí son los hombres los que se enfrentan ahí en este proceso de lucha. y si bien, la mujer también participa en estos 
procesos, finalmente no es muy visibilizada o muy reconocida su lucha en esos espacios.(...) sabemos de Guacolda, de Janequeo, sabemos de tantas lamgen que aunque invisibilizadas han estado siempre siendo parte de la resistencia de un pueblo, de los pueblos" (NTR)

Procesos políticos y espirituales que además plantean la importancia de situar y validar la diferenciación y diversidad propia de una nación en resistencia, para comprenderla efectivamente y romper la idea de homogeneización como base de una sociedad:

"Es importante no perder el sentido de contexto, lo que pasa en Santiago, que es otro contexto, otras situaciones, más bien warriache (en referencia a ciudades como Santiago u otras), no sé cómo será allá en la diáspora, pero no es lo mismo que ocurre acá, hay situaciones comunes, pero hablar desde allá para acá, de cómo estamos, cuáles son las salidas a los problemas, se pierde, porque hay situaciones que no se ven sino estando, vinculándose (...) cada territorio tiene su propia forma, al igual que sus procesos y problemas..." (NCX).

Muy asociado a estos posicionamientos, se plantea la militancia o hacerse parte de los múltiples procesos de retorno y resistencias por mantener en práctica el mapuchegen (ser mapuche):

$<$... Yo me miro, y yo creo que usted también se mira, hace diez años atrás y seguramente diez años atrás somos tan distintos a lo que somos hoy día. Porque vamos aprendiendo, porque también nos toca asumir una responsabilidad, que no se la podemos entregar a otras personas. No le podemos decir al Estado: "No! es que es su responsabilidad". Hay cosas que son nuestra responsabilidad. Entonces los niños que van surgiendo hoy día ya vienen con un orgullo, se van sintiendo orgullosos y ya vienen con otro pensamiento. $Y$ también ese pensamiento es producto del trabajo que se está haciendo ahoras (NLILI).

Por tanto, se vincula estrechamente a este proceso la politización de la sanación y el retorno al buen vivir;

"Creo que hablar eso históricamente no es justificable, no es justificar que ya nosotros venimos de una historia violenta, jes darnos cuenta de porque somos así y que podemos hacer para sacarnos todo eso! Ya, dejarlo como atrás y olvidarlo, que sea un ciclo que cierre que ya se termine. El problema es que igual esta difícil porque actualmente los gobiernos, o los estados país, por así decirlo, siguen siendo igualmente crueles y muchas veces ni siquiera el mismo estado, sino que el sistema. Nos va matando de a poco y seguimos siendo violentados. Entonces es sumamente preocupante en ese sentido" (NQR).

En cuyo ámbito las resistencias y la movilización por descolonizar y deconstruir estas dinámicas cobran lugar: 
“... me hace sentido el reclamar nomás nuestros espacios y el imponer nuestra voz. Porque de las experiencias que hay del feminismo comunitario, nadie les ha ofrecido la palabra que ellas están reclamando, se lo han tomado porque obvio que corresponde, si finalmente somos la otra mitad de las comunidades, de los colectivos. Y claro es como la determinación y el impulso de imponerse nomás siento yo, de imponer nomás los temas en las discusiones" (NMP).

Al respecto, se piensan entre otras posibilidades, la necesidad de fortalecer articulaciones de mujeres mapuche en torno a feminismos apropiados y resignificados, o bien a procesos movimentales que, sin apelar a los códigos mandatados por el colonialismo y las urbes, como es hablar necesariamente en clave feminista para validar los procesos, permitan remirar y reencontrarse con los saberes y prácticas negadas:

$<$ Yo creo que es necesario rescatarnos entre nosotras mismas. (...) Creo que es como que de alguna forma se está derrumbando un orden y que el concepto de acuerparnos es sostenernos entre todas, porque igual todas tenemos nuestros daños. Es súper difícil y agotadora la maternidad y de repente yo he visto que en una junta, en un xawün puede ser cómo un rescate para muchas mujeres, lo mismo que puede ser compartir ciertos oficios ... (NMP)

Posicionamiento en que se tejen puntos de encuentros, divergencias y heterogeneidades respecto a la apropiación o resignificación del feminismo como apuesta política para contrarrestar las violencias internalizadas:

\section{Posicionamientos políticos de mujeres mapuche: Resistencias y subversión ante el estado, los mandatos feministas hegemónicos y las violencias internas}

Los resultados de esta investigación dan cuenta de distintas trayectorias de vida enlazadas en un tronco común. Incidiendo memoria, familia, significados y costos asociados a la consecución de las autonomías personales en las diferenciaciones y énfasis que cada quién desplegó en sus posturas. Siendo cada posicionamiento, fruto del cruce de distintos aspectos como posición social, familia, cuerpo-sexualidad y pertenencia o adhesión a determinadas orgánicas sociopolíticas mapuche. Esta situación concuerda con la noción de interseccionalidad, el vínculo entre cuerpos-territorios y la diferenciación e implicación del colonialismo en el género a que refieren distintas autoras (Lugones 2008, 2011; Espinosa 2009; Segato 2011, 2018; Cumes 2012, 2014; Cabnal, 2010; Salgado 2012; entre otras).

La afectación del patriarcado colonial (Cumes, 2011) se constituye en una situación transversal a los distintos cuerpos racializados (Espinosa, 2009; Cumes, 2011) y empobrecidos. Situación inherente a los movimientos sociales de izquierda y autónomos, 
determinadas corrientes feministas que mantienen perspectivas eurocéntricas y en agendas no situadas en los contextos locales y de Abya Yala.

\section{La racialización de la violencia en contexto mapuche}

La violencia se asume como un fenómeno estructural y colateral que interviene sobre la sociedad mapuche generando sucesivas inequidades y afectaciones a la salud de las personas en su vinculación espíritus-cuerpos-territorios, a raíz del extractivismo de las empresas transnacionales, el sucesivo despojo del ixofij mogen y la evangelización como un fenómeno de violencia espiritual que se ha agudizado en las últimas décadas.

La influencia del cristianismo como parte de la empresa colonial, irrumpe en la comprensión de mundo unificándola, transversalizando los ejes del entramado colonial en las distintas estructuras sociopolíticas y culturales de los pueblos/naciones originarias, entre ello, la marcada presencia de hombres en los roles tradicionales, el ejercicio de la sexualidad, la conformación y comprensión de familia. Todo lo cual, integra la consolidación de Europa como centro y referencia del mundo y de un orden social afín a su sostenimiento.

El extractivismo, la desterritorialización, la mercantilización de los cuerpos y de todas las vidas y necesidades humanas pasan a ser constitutivas de la instauración de dicho modelo, generando mediante estos hechos una serie de violencias colaterales y transgresiones que se manifiestan en las distintas afecciones de salud de sus habitantes. Las migraciones, la reconversión de las actividades productivas junto con el tipo de megaproyectos o residuos que se instalan en los territorios, gatilla, además de una serie de efectos sobre la población como son empobrecimiento, violencia contra mujeres y otras corporalidades, la aparición de nuevas enfermedades como el $\mathrm{VIH}$ y adicciones a sustancias, entre otras (Bey, Ulbricht y Person, 2019. Además de aquellas asociadas al cuerpo de las mujeres, la economía y la soberanía alimentaria.

\section{Colonialismo internalizado y desnaturalización de las violencias internas}

En este contexto colonialista-patriarcal-capitalista en constante adaptación, el retorno no puede remitirse a un hecho material, como es volver de la diáspora al territorio histórico o ancestral, sino también a prácticas tendientes a descolonizar los sentidos, los estereotipos, y discursos hegemónicos que el mundo de la modernidad potencia especialmente en los centros urbanos, como es el caso de las ciudades capitales y los distintos pueblos o localidades que constituyen los centros cívicos de la comunas y regiones constituidas sobre territorios mapuche. Pese a estar algunos de ellos muy cercanos a los territorios en que se mantienen los lof y comunidades indígenas, mantienen un férreo contexto de discriminación y racismo respecto a los pueblos originarios y los procesos de 
movilización política. Situación que se fomenta con los medios de comunicación oficiales, los fundamentalismos religiosos, la oposición sostenida por los colonos instalados en el territorio y la violencia institucional.

Considerando las complejidades, y particularidades que gesta este proceso de irrupción en gulumapu, el retorno se constituye en un proceso aplicable a todxs sus miembros, sea que se habite los "dos mundos"22 y/o quienes siempre, o progresivamente, volvemos a vivir en $p u$ lof ${ }^{23}$. Lo cual involucra cuestionarse, desaprender, buscar y retomar desde todos los sentidos un modo de vida propio, con base en la práctica del kimün ${ }^{24}$. En cuyo caso recrear el mundo de lo propio no implica una "vuelta ciega al pasado y a las costumbres", sino a una readecuación de los elementos y significantes considerados importantes para seguir viviendo desde las epistemologías propias en un contexto globalizado y altamente tecnologizado (Pérez, 2018) y una deliberación constante de lo que se quiere ser (Cumes, 2012).

Siendo necesario para el pueblo/nación mapuche retornar a sus sentidos espirituales de vida implícitos en el mapuche feyentun y la vinculación que ésta adquiere en los cuerpos-territorios de las mujeres, en tanto constituyen una misma raíz (Cabnal, 2010). En el caso mapuche esta situación se grafica en algunos de los piam existentes respecto a la composición del mundo, como son wangülen, y xenxen y kaykay, donde no se precisa el poder en los hombres como regidores del mundo, sino en la complementariedad y cuatricidad de la vida y que sólo es posible estar bien en la medida que se está en armonía con el propio ser, la familia, el territorio, en todos los aspectos del bienestar (Marileo y Salas, 2011). Todo lo cual implica una escucha y revisión crítica de la memoria y procesos movimentales que configuran el presente de los pueblos.

\section{Autodeterminación, movimientos de mujeres mapuche y feminismos indígenas. Una trama indisociable entre lo personal y lo colectivo}

Entre los hallazgos de esta investigación se encuentra el cruce de experiencias personales con la necesidad de develar estas problemáticas y el compromiso por politizarlas a fin de modificar las realidades nocivas que se expresan en la sociedad propia. Situación que a ratos suena paradojal en el sentido que no debiese ser necesario vivir situaciones dolorosas para empatizar con ellas y politizarlas, sin embargo, la misoginia,

\footnotetext{
${ }^{22} \mathrm{En}$ referencia a la vida cotidiana que tiene lugar en el territorio o lof y las ciudades coloniales.

${ }^{23}$ Forma de organización territorial mapuche, lo integra el territorio histórico, el cual se configura a partir de un tronco común y un entorno natural y espi ritual especifico que le otorga una particularidad respecto de otros. Esta designación corresponde a una forma tradicional, que resulta de mayor complejidad y envergadura que aquello que el estado define como comunidad indígena.

${ }^{24}$ Conocimiento mapuche
} 
como otras implicaciones del sistema patriarcal, es un presente transversal a toda sociedad y ser.

Las posibilidades de vivir un az mapu distinto en lo inmediato es un proceso complejo, y permeado de distintos sentires, procesos personales y políticos que no están aislados de la contingencia militar estatal y colonial constante. Por lo que es difícil sentar las bases para un proceso distinto cuando se ha asumido determinada generización del ser zomo, como parte de la raíz cultural y el deber ser mapunche, situación que trastoca los conocimientos propios del mapuche feyentun y la importancia de ambas energías en la composición del mapuche mogen y el wajontü mapu.

La colectivización de la crianza, en este contexto, se asume como proyección y reversión de la violencia, con el fin de que las hijxs y niñxs se desenvuelvan en un contexto de deconstrucción y desnaturalización del "resistir el dolor como motor de vida", lugar en que la sanación se asuma como parte del camino de liberación. Contexto en que maternidad, aborto, parto respetado, conocimientos en torno al autocuidado de los cuerpos expresan trayectorias históricas distintas de las sociedades coloniales respecto del patriarcado como elemento fundante de sus estructuras sociales.

\section{Reflexiones finales}

La micro y macropolítica se unen en estos posicionamientos, y los torna campos que deben actuar en consonancia. Por lo que la violencia puede ser comprendida como un fenómeno con muchas aristas y un küxan (enfermedad) que al calar en lo espiritual y sociocultural solidifica la estructura patriarcal colonial, configura formas patriarcales propias y prácticas de injusticia propia.

El desarrollo de esta investigación mediante un método implicativo y descolonizador, me ha permitido evidenciar cómo se ha ido internalizando la práctica colonial patriarcal y capitalista de la "producción" académica, en los ámbitos profesionales y técnicos, y en la práctica investigativa en sí, como en quienes se hacen parte de estos procesos. Método que en el campo de los conocimientos de los pueblos indígenas y precoloniales suele adquirir un corte extractivista y unidireccional. Proceso que, como han venido planteando distintos investigadores de Abya yala y otros territorios, tiñe la práctica investigativa de múltiples formas de extractivismo, racismos y clasismos solapados, como de expresiones capitalistas.

Entre las repercusiones del colonialismo internalizado, fruto del desapego al mapuche mogen en lo cotidiano y al sentido de espiritualidad que compone la existencia y la relación cuerpo-espíritu, se asumen la asunción de la heterosexualidad como régimen colonial, el binarismo sexo género (Segato, 2011), el dualismo respecto a la vida, (vidamuerte, blanco-negro, bueno-malo, etc.), y la capitalización de la vida con el consiguiente 
empobrecimiento y/o transformación del vínculo persona-territorio, el cual se sostiene en un rol de resguardo, protección y respeto del che hacia el territorio (Marileo y Salas, 2011).

El avance nocivo de la noción de progreso del proyecto civilizatorio y sus efectos sobre la biodiversidad y el mundo social, ha generado que parte de dichas sociedades coloniales y mestizas visualicen en el modo de vida de las naciones originarias un camino posible, resignificándose, a partir de aquello, los estereotipos asentados por las lógicas del desencuentro y recreándose formas de vivir que desatienden el dinamismo de las ideologías de la modernidad. Sin embargo, su sostenimiento enfrenta enormes complejidades dada la profunda internalización de las lógicas mercantiles, de apropiación, de celeridad y de una comprensión monista de la realidad (Cumes, 2012).

El racismo, la segregación social, las múltiples formas de discriminación, el machismo, en conjunto con la serie de transgresiones que experimenta la vida social y los territorios, han calado profundo en la sociedad mapuche, y difícilmente con el contexto de violencia estructural que se incrementa y que en lo inmediato no expresa una voluntad real de cambiar, sino más bien proporciona "remedios para el propio mal que inocula" (Segato, 2011 , p. 22), reafirma la significación política espiritual atribuida a los posicionamientos de quienes participaron de este proceso, en tanto, asume que tomar acción critica en repararse y resarcir las violencias colaterales y formas patriarcales que manifiestan las orgánicas propias, es inherente a la reconstrucción de la nación mapuche.

\section{Referencias}

Bey, G. S., Ulbricht, C. M., y Person, S. D. (2019). Theories for Race and Gender Differences in Management of Social Identity-Related Stressors: a Systematic Review, Journal of Racial and Ethnic Health Disparities, 6(1), 117-132.

Cabnal, L. (2010). Acercamiento a la construcción de la propuesta de pensamiento epistémico de las mujeres indígenas feministas comunitarias de Abya Yala, Feminismos diversos: el feminismo comunitario. Acsur Las Segovias, pp. 11-25.

Calfio Montalva, M. (2012). Peküyen. En H. Nahuelpan, H. Huinca, P. Mariman, L. Cárcamo, M. Mora, J. Quidel, E. Antileo, F. Curivil, S. Huenul, J. Millalen, M. Calfío, J. Pichinao, E. Paillan y A. Cuyul, Ta iñ fijke xipa rakizuameluwün. Historia, colonialismo y resistencia desde el país Mapuche (pp. 285-304). Ediciones Comunidad de Historia Mapuche. 
Carrillo Franco, B.; Zapata Martelo, E.. y Vásquez Garcia, V. (2009). Violencia de género hacia mujeres del Frente de Pueblos en Defensa de la Tierra, Política y Cultura, (32), 127-147.

Chirapaq y Ecmia (2013). Violencias y mujeres indígenas, Documento preparado para la CSW57. http://chirapaq.org.pe/es/wpcontent/uploads/sites/3/2018/03/Violencias-y-Mujeres-Indigenas-Documentopreparado-para-la-CSW57.pdf.

Crenshaw, K. (1991). Mapping the Margins: Intersectionality, Identity Politics, and Violence against Women of Color. Stanford Law Review, 43(6), 1241-1299.

(2012). Cartografiando los márgenes. Interseccionalidad, políticas identitarias, y violencia contra las mujeres de color. En Méndez (coord.), Intersecciones: Cuerpos y sexualidades en la encrucijada (pp. 87-124). Bellaterra.

Cumes Simon, A. (2012). Mujeres indígenas, patriarcado y colonialismo: un desafío a la segregación comprensiva de las formas de dominio, Anuario Hojas de Warmi, (17), 1-16.

(2014). La "india" como "sirvienta": Servidumbre doméstica, colonialismo y patriarcado en Guatemala. [Tesis, doctorado en Antropología Social. Centro de Investigaciones y Estudios superiores en antropología social, CIESAS]. http://repositorio.ciesas.edu.mx//handle/123456789/283

Dei, G. (2003). Critical Perspectives ond indigenous research. SocialistStudies / Étudessocialistes, 9(1)

De Sousa Santos, B. (2012). Cuando los excluidos tienen Derecho: justicia indígena, plurinacionalidad e interculturalidad. En B. Sousa Santos y Grijalba (Edits.), Justicia indígena, plurinacionalidad e interculturalidad en Ecuador (pp. 13- 50). Ediciones AbyaYala. 
Espinosa Miñoso, Y. (2009). Etnocentrismo y colonialidad en los feminismos latinoamericanos: complicidades y consolidación de las hegemonías feministas en el espacio transnacional, Revista Venezolana de Estudios de la Mujer, 14(3), 37-54.

Grosfoguel, R. (2011). La descolonización del conocimiento: dialogo crítico entre la visión descolonial de Frantz Fanon y la sociología descolonial de Boaventura de Sousa Santos. En A. Vianello y B. Mané (edits.), Formas-Otras: Saber, nombrar, narrar, hacer, IV Training Seminar de jóvenes investigadores en Dinámicas Interculturales (FJIDI) (pp.97-108). CIDOB Edicions.

Hernández Castillo, R. (2001). Entre el etnocentrismo feminista y el esencialismo étnico: las mujeres indígenas y sus demandas de género, Debate Feminista, 24, 206-229.

Lugones, M. (2008). Colonialidad y género. Tabula Rasa, 9, 73-101.

(2011). Hacia un feminismo descolonial, La manzana de la discordia, 6(2), 105-119.

Marileo, Armando y Salas, R. (2011). Filosofía Occidental y Filosofía Mapuche: Iniciando un Diálogo, enlsees, (9), 119-138.

Mercado-Catriñir, X. (2011). Violencia hacia la mujer y az mapu, sistema jurídico mapuche: reflexiones y perspectivas, Nuke Mapuförlaget: Working Paper Series, (39), 151.

., Zegers Balladares, V., Tragolaf Ancalaf, A., y Contreras Rivera, C. (2015). Yamuwvn mu ka poyen tañi ixofij mogen amuleay tañi kvme mogen. Con respeto y amor por las distintas vidas, avanzamos hacia el buen vivir. (Sistematización de nvxamkawvn (encuentros de conversación) y definición de propuestas entre mujeres y organizaciones mapuche en torno a la violencia contra mujeres mapuche y aplicación de sistemas de justicia propia, Corporación de Mujeres Mapuche Aukiñko Zomo e Instituto Nacional de Derechos Humanos. 
Moreno Raiman, M. (2020). Del cuerpo-territorio como re-apropiación para re-existencias emancipatorias. Revista Reflexiones Marginales, 57.

Paredes, J., y Guzmán, A. (2014). El tejido de la rebeldía ¿Qué es el feminismo comunitario?. Bases para la despatriarcalización, Mujeres Creando Comunidad, Artes Gráficas.

Nahuelpan Moreno, H. (2013). "Las "zonas grises" de las historias mapuche. Colonialismo internalizado, marginalidad y políticas de la memoria", Revista de Historia Social y de las Mentalidades, 17(1), 11-33.

(2015). "Nos explotaron como animales y ahora quieren que no nos levantemos". Vidas despojables y micropolíticas de resistencia mapuche. En E. Antileo, L. Cárcamo-Huechante, M. Calfio y H. Huinca-Piutrin (Eds.), Amükan ka kuxankan zugu wajmapu mew. Violencias Coloniales en Wajmapu (pp. 271300). Ediciones Comunidad de Historia Mapuche

Oyěwùmí, O. (1997). Invention Of Women: Making An African Sense Of Western Gender Discourses. University of Minnesota Press.

(2017). La invención de las mujeres. Una perspectiva africana sobre los discursos occidentales del género. En la frontera.

Peña Axt, J., Huentequeo Toledo, M., \& Cayupan Constanzo, S. (2018). Relatos de mujeres mapuche de la araucanía: revisitación a las formas de familia bajo la figura de la bigamia. Tabula Rasa, (28), 323-346 https://doi.org/10.25058/20112742.n28.14

Pérez Moreno, M. (2018). O’tanil. Staleltseltaletik. Una apuesta por un conocimiento propio desde los pueblos originarios. En Leiva et al., (auts.), Prácticas otras de conocimiento(s): Entre crisis, entre guerras (pp. 429- 450). Taller Editorial La Casa del Mago. 
Pichinao Huenchuleo, J. (2015). La mercantilización del mapuche mapu (tierras mapuche). Hacia la expoliación absoluta. En E. Antileo, L. Cárcamo-Huechante, M. Calfio y H. Huinca-Piutrin (Eds.), Amükan ka kuxankan zugu wajmapu mew. Violencias Coloniales en Wajmapu (pp. 87-106). Ediciones Comunidad de Historia Mapuche

Quiñimil Vásquez, D. (2012) PETU MONGENLEIÑ, PETU MAPUCHENGEN. Todavía estamos vivxs, todavía somos mapuche. Un proceso autoetnográfico para la descolonización feminista de las categorías mujer, mapuche, urbana, a través del aborto [Tesis de Máster, Universidad de Granada]. http://hdl.handle.net/10481/22726

Rivera Cusicanqui, S. (2010). Ch'ixinakaxutxiwa Una reflexión sobre prácticas y discursos descolonizadores. Tinta Limón.

Sánchez, M. (2016). Espiritualidad Indígena y participación femenina, Lectora: Revista de dones i textualitat, 22, 59-65.

Salgado, J. (2012). El reto de la igualdad: género y justicia indígena. En De Sousa Santos y Grijalba (Edits.), Justicia indígena, Pluriculturalidad e interculturalidad en Ecuador (pp. 243-278). Fundación Rosa Luxemburg/AbyaYala.

Segato, R. (2011). Género y colonialidad: en busca de claves de lectura y de un vocabulario estratégico descolonial, En K. Bidaseca y V. Vásquez (Comps.), Feminismos y poscolonialidad. Descolonizando el feminismo desde y en América Latina (pp. 17-30). Godot.

(2018). Contra-pedagogías de la crueldad. Prometeo Libros.

Shiva, V. (1995). Abrazar la vida: mujer, ecología y pervivencia. Horas y HORAS.

Vásquez, A. (2015). Expedientes del dolor: mujeres mapuche en la frontera de la violencia (1900-1959). En E. Antileo, L. Cárcamo-Huechante, M. Calfio y H. HuincaPiutrin (Eds.), Amükan ka kuxankan zugu wajmapu mew. Violencias Coloniales en Wajmapu (pp. 141-157). Ediciones Comunidad de Historia Mapuche 